\title{
Groundbased investigation of asteroid 9969 Braille, target of the spacecraft mission Deep Space $1^{\star}$
}

\author{
M. Lazzarin ${ }^{1}$, S. Fornasier ${ }^{1}$, M. A. Barucci ${ }^{2}$, and M. Birlan ${ }^{2}$ \\ 1 Dip. di Astronomia, Vic. Osservatorio 5, 35122 Padova, Italy \\ 2 Obs. de Paris, 92195 Meudon Principal Cedex, France
}

Received 19 December 2000 / Accepted 24 May 2001

\begin{abstract}
Asteroid 9969 Braille (1992 KD) was encountered on July 29, 1999 by the Deep Space 1 mission, the first of NASA's New Millennium Program, launched on October 24 1998. The data obtained by the space mission seem to indicate a composition of the object similar to that of Vesta. To complete the information obtained in the infrared region by the Deep Space 1 mission we have performed a visible spectroscopic and photometric investigation of the asteroid respectively with the $1.5 \mathrm{~m}$ telescope and the NTT of ESO, La Silla. The spectrum was obtained in the spectral range 4500-8200 $\AA$ and, for the photometry, $B V R I$ filters were used. In this paper we report the results of the analysis of the data obtained indicating that, on the basis of our visible data, the composition of the asteroid may range from V-type to Q-type, but we observe also a strong similarity to the H-type ordinary chondrites.
\end{abstract}

Key words. solar system: general - minor planets - asteroids

\section{Introduction}

In the last years, the number of space missions devoted to the investigation of comets and asteroids has increased due to the importance of studying these small bodies for the understanding of the origin and evolution of our Solar System. In fact, comets and asteroids are believed to be the most primitive objects in the Solar System and they hold many clues about the physical, thermal and compositional conditions present during the formation of the Solar System. In particular, the investigation of the composition of these bodies can give fundamental information on these subjects. In this context, we have made a spectroscopic and photometric study of the asteroid 9969 Braille (1992 KD), a target of the Deep Space 1 mission.

The Deep Space 1 is the first of a series of technology demonstration probes being developed by NASA's New Millennium Program to test new technologies for future space and Earth-observing missions. The spacecraft made

Send offprint requests to: M. Lazzarin,

e-mail: lazzarin@pd.astro.it

* Based on observations carried out at the European Southern Observatory (ESO) of La Silla, Chile, programs N.62S-0173 and N.62S-0305A. a fly-by with the asteroid 9969 Braille (formerly known as 1992 KD) on 29 July 1999. Just after the encounter with Braille, NASA decided to extend the DS1 mission to flyby the dormant comet Wilson-Harrington in January 2001 and comet Borrelly in September 2001.

Along with the technology demonstrations, the probe carries the Miniature Integrated Camera-Spectrometer (MICAS), an instrument combining two visible imaging channels with UV and IR spectrometers. MICAS was used to study the chemical composition, geomorphology, size, spin-state, and atmosphere of 9969 Braille (Soderblom et al. 1999). DS1 carries also the Plasma Experiment for Planetary Exploration (PEPE), an ion and electron spectrometer which is able to measure the solar wind during cruise, the interaction of the solar wind with the target bodies during encounters, and the composition of the cometary coma.

Deep Space 1 arrived within $26 \mathrm{~km}$ of asteroid 9969 Braille at 04:46 UT on July 29, 1999. The asteroid was not successfully imaged during the close flyby due to an on-board target-tracking problem. Two images were obtained 914 and $932 \mathrm{~s}$ after the closest approach from about $14000 \mathrm{~km}$ and a dozen infrared spectra were 
obtained about 3 minutes later, revealing what is probably a V-type classification of this NEA (Soderblom et al. 1999). The images show that 9969 Braille has an irregular shape, and is approximately $2.2 \mathrm{~km}$ along its longest side and $1 \mathrm{~km}$ at its shortest.

The study of Braille is also interesting in itself: it was discovered by E. F. Helin in 1992 (Helin et al. 1992) and very little was known about it before the encounter with the spacecraft. It was discovered as an Amor asteroid and a successive more accurate determination of the orbit classified it as a Mars crosser. The study of these objects is important because they represent one of the most peculiar classes of objects in the Solar System and their origin is not well understood yet. This population is very diverse in nature, many having unusual shapes (very elongated or bifurcated); binary systems have been discovered among the population. Some of these objects have a complex, nonprincipal axis rotation state while some others display very long rotational periods, which are not easily explained by the current dynamical and collisional models.

Their diversity is also reflected by the different taxonomic types present in the population: S, C, V, M, Q and $\mathrm{D}$ types have been identified to date (Binzel et al. 1997).

The origin of these bodies also is not well understood. We know that their present orbits are unstable over time scales much shorter than the age of the Solar System, and that over these time scales NEAs are ejected or collide with the Sun or a planet. Because the cratering record on the Moon suggests a fairly constant NEA population during the past 2 billion years, new NEAs must be continuously supplied by some sources in order to maintain the present steady state. Currently, two sources for NEAs have been identified. The first one is the Main Belt, where gravitational perturbations by the major planets cause dynamical resonances which provide escape routes. The second source is represented by extinct comets. A good number of NEAs represent the final evolutionary state of comets, that is, a devolatilized nucleus. An example is the asteroid Apollo 4015, the next target of the DS1 mission, which was the comet Wilson Harrington in 1949. It is not clear, however, what the efficiency of the delivery mechanism is, and what percentage of the NEAs we observe today comes from the one or other source. One way of addressing this problem is to characterize these objects spectroscopically, in order to derive their mineralogy.

Another interpretation of the origin of Braille, theorized by some astronomers, is that it may have been knocked off of Vesta and in effect the data obtained from the DS1 present on the WEB site of the DS1 at JPL would suggest a V-type composition.

Also Hicks et al. (1999) investigated Braille obtaining $B V R I$ photometric data: they find colors closer to those of ordinary chondrite and basaltic achondrite meteorites than those of the vast majority of main-belt asteroids.

Binzel et al. (2001) also made a spectrum of Braille in the visible region suggesting a similarity to Q-type asteroids (A. W. Harris, private communication).
We have spectroscopically and photometrically investigated 9969 Braille in order to obtain information on its surface composition and its possible variations and in particular to complete the data obtained in the infrared region by the DS1 mission. A spectrum between about 4500 and $8200 \AA$ and $B V R I$ data have been compared; unfortunately, observations planned in the near infrared have not been successfull owing to bad weather conditions.

\section{Observations and data reduction}

Spectroscopy: we performed a spectroscopic investigation of 9969 Braille on 17 March 1999 at the European Southern Observatory of La Silla (Chile), with the $1.5 \mathrm{~m}$ telescope equipped with a Boller \& Chivens spectrograph and a Loral Lesser CCD as detector $(2048 \times 2048$ pixels $)$. The grating used was a $225 \mathrm{gr} / \mathrm{mm}$, with a dispersion of $331 \AA / \mathrm{mm}$ in the first order. The CCD has a $15 \mu \mathrm{m}$ square pixels, giving a dispersion of about $5 \AA /$ pixel in the wavelength direction. The spectral range is about $0.45<\lambda<$ $0.82 \mu \mathrm{m}$ with a FWHM of about $10 \AA$.

The spectrum was recorded through a slit oriented in the East-West direction. The slit was opened to about 8 arcsec in order to reduce effects due to differential refraction and the possibility of losing signal due to guiding errors of the telescopes.

9969 Braille was observed on 17 March 1999, at 01:30:58 UT, with an exposure time of 75 min. During the observation, Braille had a visual magnitude of about 18.3, and an airmass of about 1.1.

We also recorded bias, flat-field, calibration lamp spectra, and solar analog stars' spectra at different intervals throughout the same night.

The solar analog stars observed (Hyades64, HD 44594, HD 89010, HR 6060, HD 144585, HD 76151) (Hardorp 1978), are necessary to remove the solar contribution from the spectrum of the asteroid and to obtain the asteroidal reflectivity. The spectral behaviour of the stars was very similar during the night with differences in the reflectivity gradient around $1 \% / 10^{3} \AA$. To obtain the asteroid spectrum reflectivity we then divided the spectrum of the asteroid by the spectrum of the solar analog with airmass closest to that of the asteroid (Hyades64).

The spectrum was reduced using ordinary procedures of data reduction (Luu \& Jewitt 1990) with the software packages Midas and IDL.

These procedures include: subtraction of the bias from the raw data, division by flat field, cosmic ray removal, background subtraction, collapsing the two dimensional spectra, wavelength calibration, atmospheric extinction correction. Wavelength calibration was made using a lamp with $\mathrm{He}, \mathrm{Ar}, \mathrm{Fe}$ and $\mathrm{Ne}$ emission lines. The residuals of the wavelength calibration were $\leq 2 \AA$. After these procedures, we normalized all the spectra, both of asteroids and solar analog stars, at 1 around $5500 \AA$.

The spectrum was then smoothed with a median filter technique. Some spurious, easily recognizable, features 
Table 1. Color indices with relative errors of Braille. Two obervations were performed during the 13-14 night: at 1:36 UT and $5.51 \mathrm{UT}$.

\begin{tabular}{|c|c|c|c|}
\hline \hline DATE & $B-V$ & $V-R$ & $V-I$ \\
\hline $13-14$ Feb. & $0.791 \pm 0.030$ & $0.411 \pm 0.013$ & $0.708 \pm 0.018$ \\
$13-14$ Feb. & $0.813 \pm 0.032$ & $0.441 \pm 0.010$ & $0.708 \pm 0.015$ \\
$14-15$ Feb. & $0.817 \pm 0.045$ & $0.468 \pm 0.011$ & $0.728 \pm 0.020$ \\
\hline
\end{tabular}

due to an incomplete removal of sky lines (in particular of the water telluric bands around $7200 \AA$ and of the $\mathrm{O}_{2} \mathrm{~A}$ band around $7600 \AA$ ) are present on the asteroid spectrum. The spectrum of Braille is reported in Fig. 1.

Photometry: the broadband color data were obtained at NTT of ESO on two nights (February 14-15, 1999). The SUSI2 CCD camera $(55 \times 55)$ at the $\mathrm{f} / 11$ Nasmyth focus of the $3.5 \mathrm{~m}$ New Technology Telescope (NTT) was used to obtain direct images. The EEV camera (ESO No. 46) was selected for our observations with the Bessel $B, V$, $R$, and $I$ filters (central wavelength at $0.421,0.544,0.642$ and $0.795 \mu \mathrm{m}$ respectively). The observations were carried out in $2 \times 2$ binning mode, yielding a pixel scale of $0.16 \mathrm{arcsec} / \mathrm{pixel}$. The seeing throughout the run was in the range $0.6-1.5$ arcsec. The exposure time was $90 \mathrm{~s}$.

The CCD images were reduced and calibrated with a standard method using aperture photometry (MAGNITUDE/CIRCLE in MIDAS). First, bias and flatfield corrections were performed. The instrumental magnitudes were measured using aperture photometry. The radius of the aperture used was typically about twice the average seeing. This was deemed optimum since it is large enough to include most of the point spread function, yet small enough to minimize background sky noise. Sky subtraction was performed using a 7-12 pixels wide annulus around the asteroid or reference star. Finally, for magnitude calibration, observations of standard stars were obtained over a wide range of airmasses and stellar types (three fields PG0942, SA107, and SA95 were used, Landolt 1992). The zero point, extinction and colour terms obtained from the Landolt fields were then used to convert instrumental magnitudes to apparent magnitudes. The errors quoted take into account both the instrumental errors given by photon statistics alone and the calibration error. The latter error came from the scatter in the field star photometry.

\section{Results and discussion}

$B V R I$ band images were obtained at NTT on February 14 to 15,1999 . The $B-V, V-R$ and $V-I$ colors are reported in Table 1.

Little variation on the photometric data could be attributed to surface composition variations of the object.

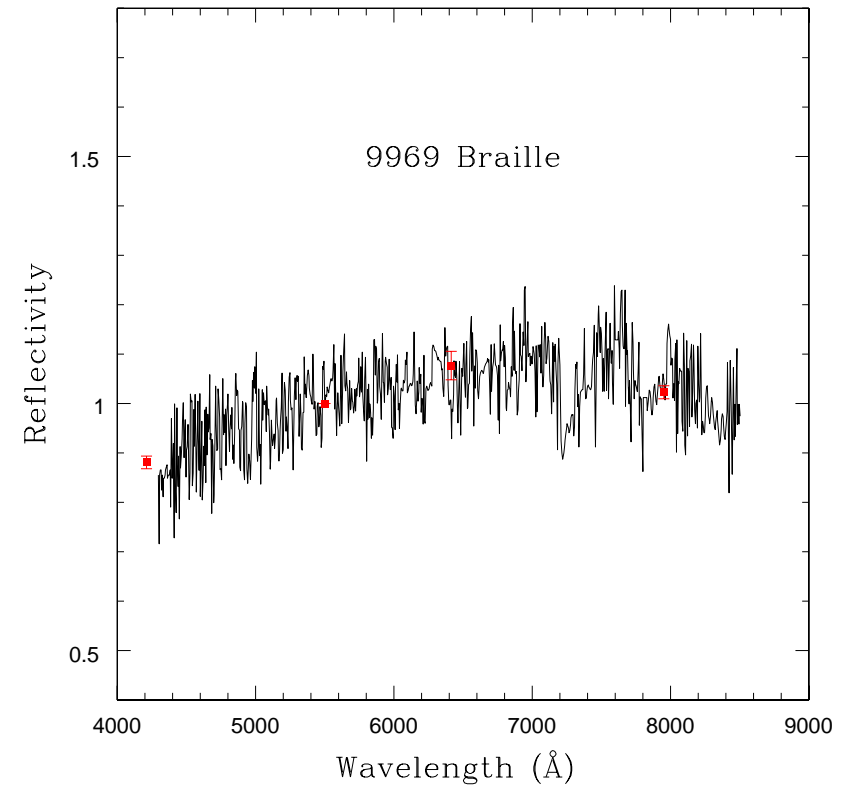

Fig. 1. Reflectance spectrum of 9969 Braille obtained on 17 March 1999 at ESO-La Silla. The spectrum is normalized at 1 around $5500 \AA$. The average broadband color data with the relative errors are also reported (squares) on the spectrum.

The spectrum of 9969 Braille (Fig. 1) has a reflectance slope of $(7.13 \pm 0.29) \% / 10^{3} \AA$ computed in the spectral range of $4300-7200 \AA$. It also presents some spurious features due to an imperfect subtraction of sky absorption bands (for example the water telluric band around $7200 \AA)$.

The average relative reflectance obtained from the $B V R I$ color photometry is also plotted in Fig. 1. The reflectivity from the photometric data was computed using solar colors. We adopted the maximum semi-dispersion as the error bar. The broadband colors agree quite well with the spectroscopic data.

In order to characterize the spectral type of 9969 Braille we used the extension of the G-mode multivariate method (Fulchignoni et al. 2000). The taxonomic classes proposed by Birlan et al. (1996) were taken into account. The results of our analysis reveal that 9969 Braille could be a V or SV asteroid. However, the spectrum of Braille does not cover the whole spectral interval of the defined classification; our analysis underlines the affinity of the asteroid to a given taxonomic class.

In Fig. 2 we compare the spectrum of Braille with that of Vesta and the result is a good match.

However, we also tried a comparison with the spectrum of a Q-type asteroid (1862 Apollo), with the average spectrum of V-type and S-type asteroids (Bus 1999) and with the spectra of several H, L and LL ordinary chondrites. What results is that in effect Braille is similar to Vesta, and compatible, within the errors, with the average of V-type asteroids, but also with the Q-type spectrum (Fig. 2). Moreover, the comparison with ordinary 


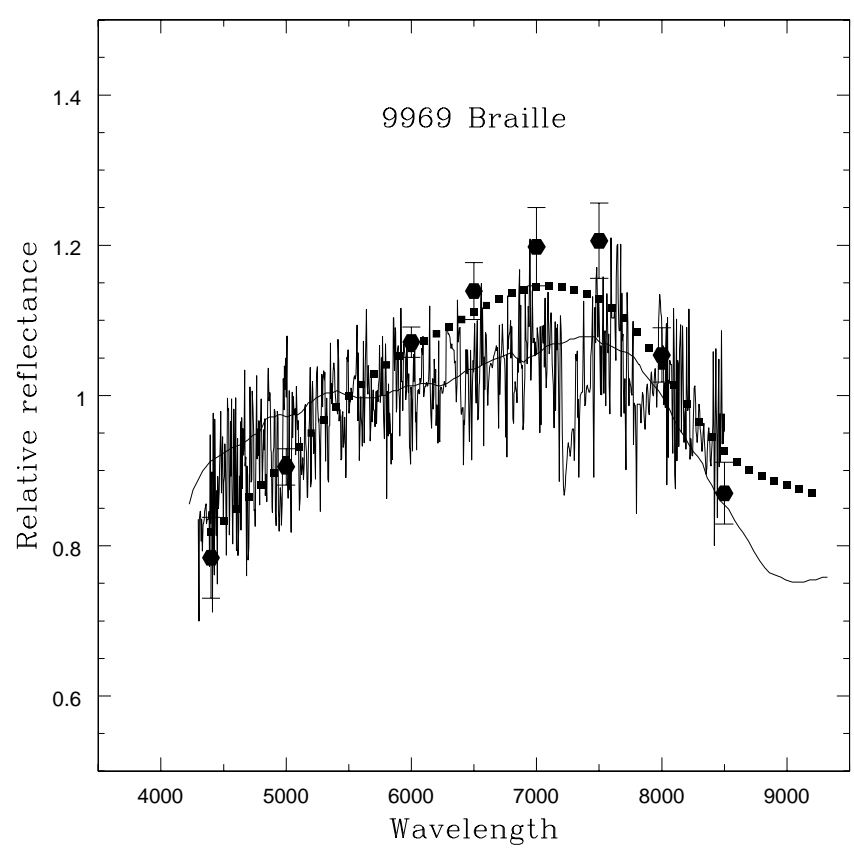

Fig. 2. Spectrum of 9969 Braille superimposed on the spectrum of asteroid Vesta (continuum) (Binzel et al. 1993), the average spectrum of $\mathrm{V}$-type (hexagons) and the spectrum of the Q-type asteroid 1862 Apollo (squares) (Bus 1999).

chondrites has revealed that the spectrum of Braille is pratically indistinguishable from that of $\mathrm{H} 4$ and $\mathrm{H} 52$ ordinary chondrites (Fig. 3). Instead, our spectrum is incompatible with that of the average spectrum of S-type asteroids (redder than the spectrum of Braille).

So, from our data we might confirm the data obtained in the infrared region by the Camera-Spectrometer MICAS of the Deep Space 1 during its fly-by with Braille on 29 July 1999 and reported by Soderblom et al. (1999). However, on the basis of our visible spectrum of Braille other interpretations of its composition are also possible: in fact it ranges between the Q-type and V-type and it shows a strong similarity with the ordinary chondrites $\mathrm{H} 4$ and H52 (Fig. 3).

\section{Conclusion}

Asteroid 9969 Braille was spectroscopically and photometrically observed during two observational runs at ESO with the $1.5 \mathrm{~m}$ telescope and the NTT respectively. From the data obtained here a clear conclusion on the composition of Braille is not easy to draw as the spectrum is compatible with that of Vesta, but also with the Q-types.

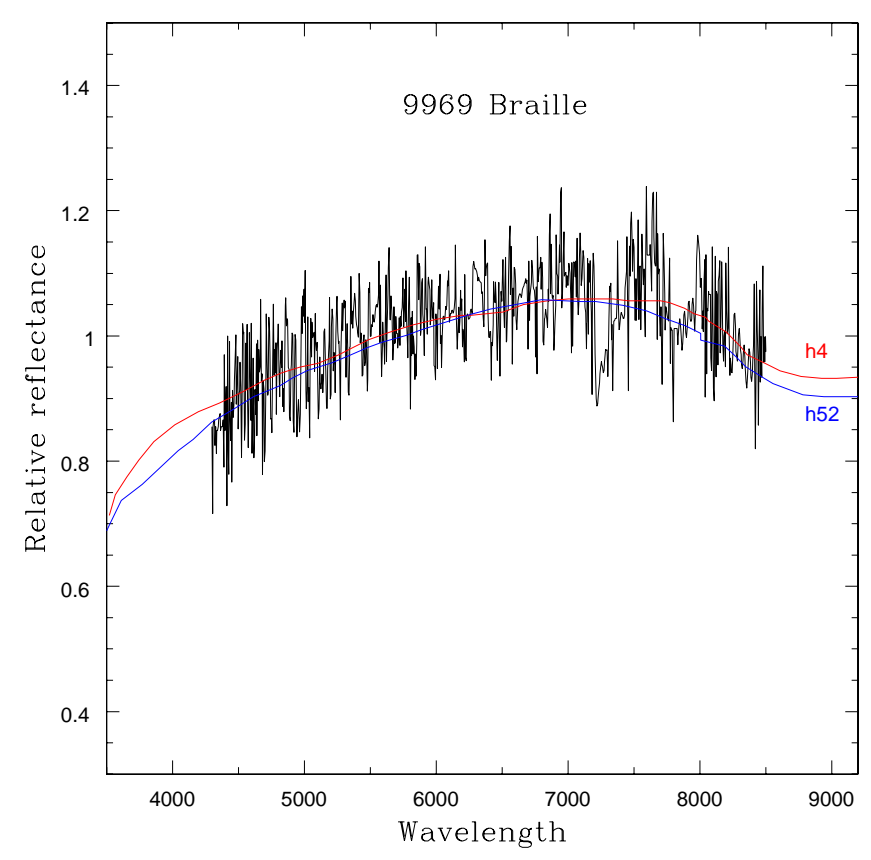

Fig. 3. Spectrum of 9969 Braille superimposed on the spectra of ordinary chodrites H4 and H52.

A strong similarity is in particular evident with the ordinary chondrites. So we think that further observations, in particular in the near infrared, would help to define the surface composition of Braille.

\section{References}

Binzel, R. P., Harris, A. W., Bus, S. J., \& Burbine, T. H. 2001, Icarus, in press

Binzel, R. P., Bus, S. J., \& Burbine, T. H. 1997, Am. Astron. Soc., DPS Meeting, BAAS, 29, 05.01

Binzel, R. P., \& Xu, S. 1993, Science, 260, 186

Birlan, M., Fulchignoni, M., \& Barucci, M. A. 1996, Icarus, 124,352

Bus, S. J. 1999, Ph.D. Thesis

Fulchignoni, M., Birlan, M., \& Barucci, M. A. 2000, Icarus, 146, 204

Hardorp, J. 1978, A\&A, 63, 383

Helin, E. F., Lawrence, K., Rose, P., Alu, J., \& Williams, G. V. 1992, IAUC 5531

Hicks, M. D., Buratti, B. J., Rabinowitz, D. L., et al. 1999, 30th Annual Lunar and Planetary Science Conference, Houston, TX, abstract, No. 1719

Landolt, A. U. 1992, Icarus, 72, 304

Luu, J. X., \& Jewitt, D. C. 1990, AJ, 99, 1985

Soderblom, L., Boice, D., Britt, D., et al. 1999, Am. Astron. Soc., DPS Meeting, BAAS, 31, 34.03 